\title{
Power strategy in DC/DC converters to increase efficiency of electrical stimulators
}

\author{
Pablo Aqueveque (1), Vicente Acuña (2), Francisco Saavedra (1), Adrien Debelle (2), \\ Laurent Lonys (2), Nicolas Julémont (2), François Huberland (2), Carmen Godfraind \\ (2), Antoine Nonclercq (2)
}

\section{(1) Universidad de Concepción, Chile; (2) Université Libre de Bruxelles, Brussels, Belgium.}

This article is distributed under the terms of the Creative Commons Attribution Noncommercial License (CC BY-NC 4.0) which permits any noncommercial use, distribution, and reproduction in any medium, provided the original author(s) and source are credited.

\begin{abstract}
Power efficiency is critical for electrical stimulators. Battery life of wearable stimulators and wireless power transmission in implanted systems are common limiting factors. Boost DC/DC converters are typically needed to increase the supply voltage of the output stage. Traditionally, boost $\mathrm{DC} / \mathrm{DC}$ converters are used with fast control to regulate the supply voltage of the output. However, since stimulators are acting as current sources, such voltage regulation is not needed. Banking on this, this paper presents a DC/DC conversion strategy aiming to increase power efficiency. It compares, in terms of efficiency, the traditional use of boost converters to two alternatives that could be implemented in future hardware designs.
\end{abstract}

Key Words: power strategy, implanted stimulator, wearable stimulator, high efficiency

Eur J Transl Myol 2016; 26 (3): 223-228

P ower efficiency is critical for electrical stimulators. Battery life of wearable stimulators and wireless power transmission in implanted systems are common limiting factors. These systems deliver large amount of power (i.e. stimulating current to the load) with a restricted power source ${ }^{1}$. Designers must therefore pay careful attention to power efficiency. Boost DC/DC converters are typically needed to increase the supply voltage of the output stage. Indeed, the supply voltage is governed by the stimulating current and by the load impedance, and is typically way larger than the voltage provided by the battery ${ }^{2}$. Most of the implant power goes through the $\mathrm{DC} / \mathrm{DC}$ converter, and it is therefore subject to high power losses. Traditionally, boost DC/DC converters are used to regulate the supply voltage of the output stage as fast as possible to a fixed value. However, since a stimulator is acting as a current source, the supply voltage of the output stage does not need to be kept to a fixed value. It only needs to be kept high enough to provide the stimulating current to the load while not saturating. Banking on this, this paper presents a DC/DC conversion strategy applied to stimulators aiming to increase power efficiency. It compares in terms of efficiency the traditional use of boost converters to two alternatives that could be implemented in future hardware designs.

\section{Materials and Methods}

Fig. 1. shows the block diagram of a typical embedded stimulator. It consists of a battery cell, a microcontroller (for circuit synchronization), a voltage boost (to raise the battery voltage to a level usable by the output stage) and the output stage. The output stage is mainly composed of a current source that delivers the required (fixed) current to the load when stimulating. It is typically based on an operational amplifier driving the gate of a MOSFET. Boost DC/DC converters are usually needed to increase the supply voltage of the output stage. Since most of the implant power goes through the DC/DC converter, its power strategy has a large impact on efficiency. Three power strategies are analyzed in this work:

- Power strategy 1 (PS1): the boost converter is switched on around the stimulation period and is set up to drive the supply voltage quickly to the desired value (traditional power strategy);

- Power strategy 2 (PS2): the boost converter is switched on between the stimulation period and is set up to drive the supply voltage quickly to the desired value;

- Power strategy 3 (PS3): the boost converter is switched on between the stimulation periods and is set up to drive the supply voltage slowly to the desired value. 


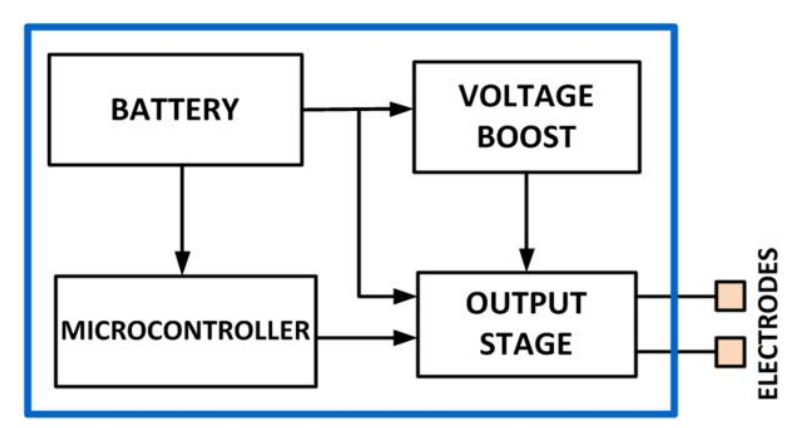

Fig 1. Block diagram of the embedded system.

Fig. 2. compares the on/off periods of the three power strategies (stimulation period is also provided).

For the traditional power supply strategy (PS1), stimulation occurs when the boost converter is enabled. The voltage regulation of the boost is set up in such a way that the supply voltage reaches its nominal value quickly. Conceptually, the voltage supply and the current delivered to the load are regulated, respectively by the boost converter and the current source, whereas only the delivered current should be. The supply voltage of the output stage only needs to be kept high enough to provide the stimulating current to the load while not saturating. Banking on this consideration, two additional power strategies are proposed (PS2 and PS3). The idea is based on the principle of a charge pump. The boost is charging a storage capacitor when the implant is not stimulating. Stimulation therefore occurs after the boost converter is enabled (see Fig. 2). The charging period of the capacitor may be fast (PS2). However, the time between two stimulations is, in many biomedical applications, quite large. The charging period of the capacitor may therefore also be slow (PS3). The schematic of the DC/DC converter is shown in Fig. 3. It is a regulated switch-mode DC power supply, converting the unregulated low DC input voltage into a controlled high DC output at a desired voltage level. A step-up (boost) converter is used to increase the input voltage. When the switch is on, the diode is reversed biased, thus isolating the voltage output. The load is supplied by the capacitor and the input voltage source supplies energy to the inductor. When the switch is off, the output stage receives energy from the inductor and from the input voltage source, charging the capacitor and supplying the load ${ }^{3}$. The boost converter is used in discontinuous mode: in each commutation cycle, the current in the inductor drops to zero (i.e. the inductor is completely discharged) during a portion of the period. This is common for loads with low power consumption. There are only two differences in the way these three strategies are implemented: the on/off periods of each DC/DC converter (see Fig. 2) and the control strategy used. In PS1 and PS2, a PI control is implemented to drive the output voltage quickly to the desired value. In PS3, the PI control is replaced by an On-Off control that

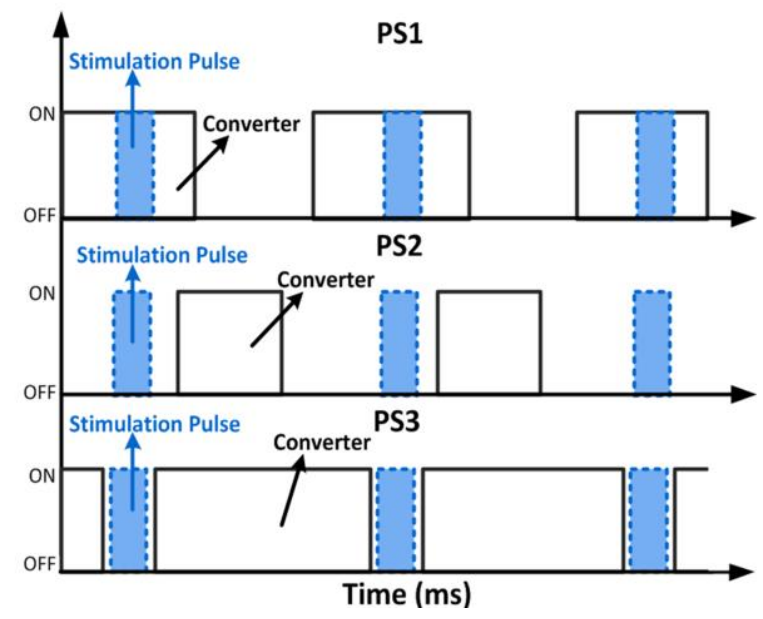

Fig 2 ON/OFF periods of the three power strategies. In PS1, stimulation occurs when the boost converter is enabled. In PS2 and PS3, it occurs after.

enables the converter during reduced time intervals, therefore charging the storage capacitor slowly. The value of the capacitor was adjusted so that the voltage drop produced by the stimulation pulse (and the capacitor discharge) is not too large, i.e. so that the supply voltage is large enough to avoid saturation. Roughly, it would occur when the supply voltage is lower than the product of the stimulation current and the impedance of the load. Since the stimulation energy needed for one stimulation pulse is known, the voltage drop on the capacitor may be calculated. As a rule of thumb, the value of the capacitance was chosen so that it can store ten times more energy than required during one stimulation pulse.

\section{Case studies}

Two case studies were chosen to illustrate the effects of these power strategies with concrete examples. The first corresponds to a portable foot drop stimulation system, developed at the Universidad de Concepción, Chile. Drop foot is the gait disturbance common in patients with stroke, multiple sclerosis, spinal cord injury and spastic cerebral palsy ${ }^{4}$. This disorder is characterized in that the person has no voluntary control of dorsiflexor muscles, which mean that it is difficult to point toes toward the body (dorsiflexion) or rotate the foot inward or outward (inversion and eversion) which carries a poor motion. The person will have serious difficulties because the front of the foot drops to the floor before the heel comes in contact with the ground, which can cause tripping or falling. Furthermore, the foot will not have the stability provided by the dorsiflexor muscles. All this increases significantly the person's energy consumption because it takes more effort to walk, with positions that are not physiological. Along with the loss of mobility, the person can be accompanied by pain and weakness. Portable foot drop stimulators must be 


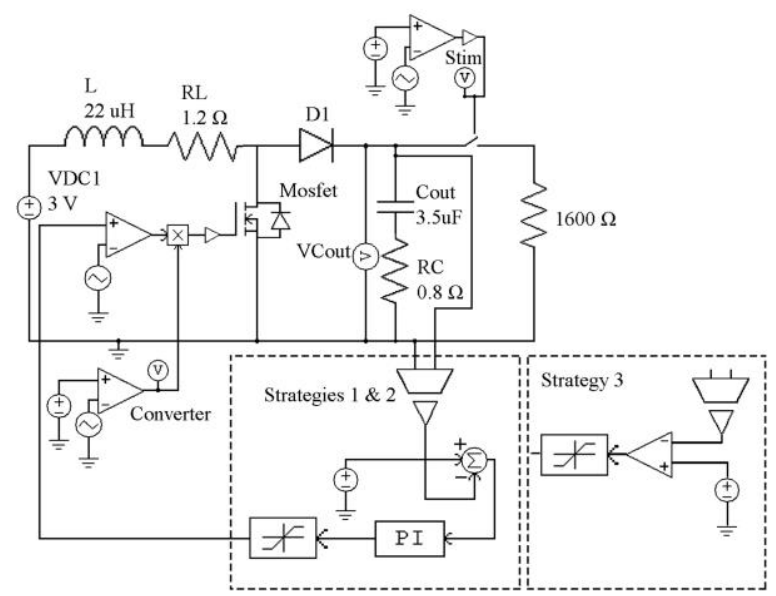

Fig 3 Schematic of the DC/DC converter. Specificities of the three strategies are displayed.

battery powered. In general, any portable device uses 2 AA batteries $(2 \times 1.2 \mathrm{~V})$ or a $9 \mathrm{~V}$ battery. Thus, to generate $100 \mathrm{~V}$ or more from the batteries, the efficiency of the booster circuits of the stimulator must be analyzed. The second case study corresponds to an implanted gastrostimulator, aiming to produce a feeling of satiety, and hence to fight obesity ${ }^{5}$. In recent years, obesity has literally reached epidemic proportions throughout the world and is now in the top three of mortal diseases. Obesity represents a major risk of health issues including cardiovascular diseases (mainly heart disease and stroke), diabetes, musculoskeletal disorders (especially osteoarthritis) and cancers (endometrial, breast, and colon) ${ }^{6,8}$. Bariatric surgery, mainly recommended to patients with a body mass index (BMI) ranging between 35 and 50, is one of the most common techniques used to induce weight loss 9 . Although effective, it suffers from important drawbacks such as its considerable costs and invasiveness, as well as long-term weight regain ${ }^{10}$. Gastric electrical stimulation (GES) is a recent technique that uses an implanted device (gastrostimulator) to stimulate the stomach, aiming to produce a feeling of satiety for obese patients, and hence to fight obesity. It has recently shown promising effects in treating obesity and could potentially overcome most of the drawbacks of bariatric surgery, being less invasive, reversible and cost effective $^{11}$. However, current gastrostimulators are bulky and are implanted by multi-incision laparoscopy, a relatively expensive and invasive procedure. This project aims to implant the device through a less invasive procedure. Attempts covered fully endoscopically procedure and, more recently, the team is focusing on single incision laparoscopic procedure.

Both proposed systems have different power requirements. Table 1 shows the major characteristics of these two stimulators. Note that stimulation frequency and stimulation pulse width are typical ${ }^{12}$. The switching frequency was adjusted to allow output voltage

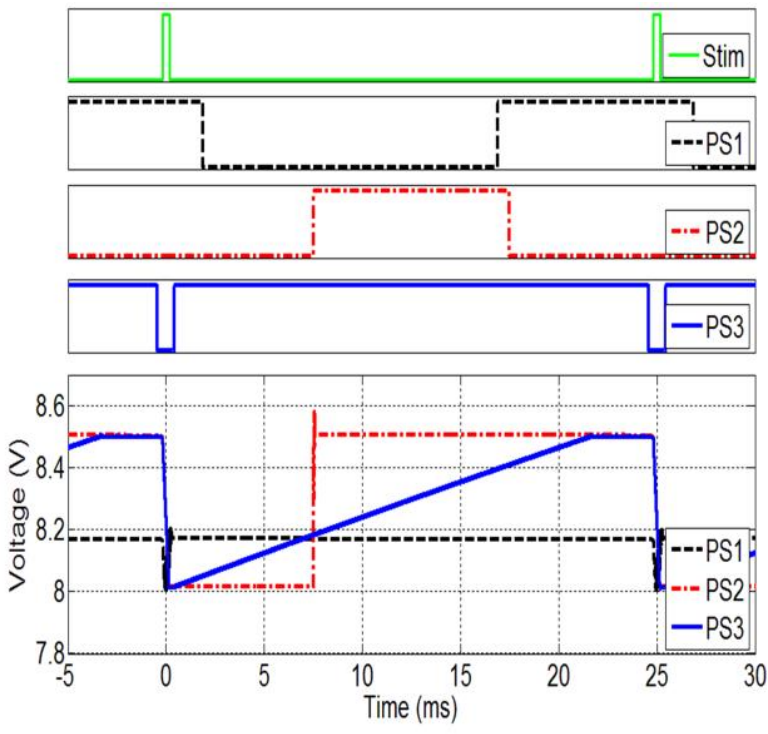

Fig 4. Comparison between strategies proposed. Top to bottom: stimulation pulse, on/off periods of the three power strategies and voltage in the storage capacitor.

stabilization before the end of the "on period" of the converter in PS1 (see Fig. 2), respectively to $50 \mathrm{kHz}$ for the drop foot stimulator and to $200 \mathrm{kHz}$ for the gastrostimulator. A high voltage MOSFET (IRF 540) was used for the drop foot stimulator and a small size one (LT3564) for the gastrostimulator.

\section{Results}

The three strategies were simulated with PSIM for both case studies. Illustrations are given in the case of the gastrostimulator and can be easily generalized for the drop foot stimulator. Efficiencies are given for both case studies.

Fig. 4 shows the voltage at the storage capacitor for the three strategies, as well as the stimulation period and the boost enable period. One can see that the voltage at the capacitor is always higher than $8 \mathrm{~V}$, as required, for every strategy. This way, saturation does not occur and fixed stimulation current is provided to the load.

Major power losses are produced by the internal resistance of the inductor and the MOSFET of the booster. Fig. 5 and Fig. 6 respectively show the current in the inductor and in the MOSFET. In PS1 and PS2, switching only occurs during a short period of time, and so do the currents flowing in the inductor and the MOSFET. Unfortunately, the values of these currents are large, which produces considerable power losses.

In PS3, switching occurs during a larger period of time, but the current that flows in the inductor and in the MOSFET is smaller. Since the charge fed to the capacitor corresponds to the charge needed by the output stage during stimulation, it is constant in all power strategies. Therefore the product of the current amplitude and the switching period is roughly constant. 

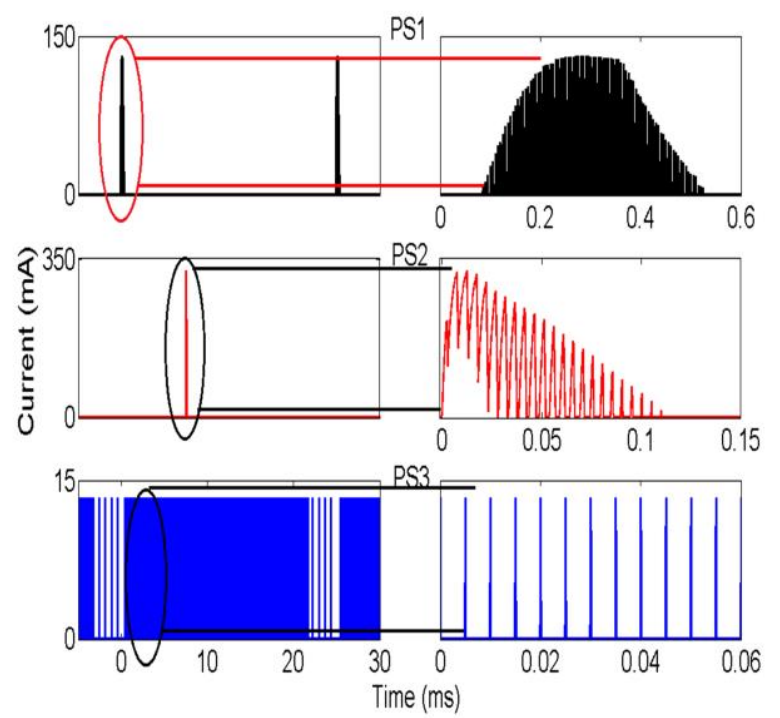

Fig 5. Current in the inductor in each strategy. Left: global picture; right: zoom on the switching period. Note that the maximum scale for PS3 is only $15 \mathrm{~mA}$, compared to $150 \mathrm{~mA}$ for PS1 and $350 \mathrm{~mA}$ for PS2.

Here, the switching period of PS3 is at least ten times longer than for PS1 and PS2, and therefore the value of the switching current is at least ten times smaller. Power losses - both in the inductor and in the MOSFET - are proportional to the square of the current amplitude. Therefore PS3 is more attractive: Roughly, power losses occur during a period of time ten times larger than PS1 and PS2, but with an instantaneous value that is a hundred times (the square of ten) lower than for PS1 and PS2. Altogether, we expect a power loss reduced by a factor of ten.

Table 2 shows the efficiency of each strategy for both case studies. It was calculated as the ratio of the power delivered to the load divided by the input power (respectively $1.6 \mathrm{k} \Omega$ and VDC1 in Fig. 3). The efficiency of PS3 is higher than the traditional power strategy (PS1) for both case studies $(11.1 \%$ higher for the gastrostimulator and $21.2 \%$ for the drop foot stimulator). The efficiency of PS2 is equal to or even lower than PS1, because the time used to recharge the storage capacitor is equal to or even lower than the one of PS1 (see Fig. 5 and 6), depending on the characteristics of the stimulator.

\section{Discussion}

This paper presents a DC/DC conversion strategy, designed for biomedical stimulators, that increases the power efficiency - in our cases by $11.1 \%$ and $21.2 \%-$ compared to traditional designs. The resulting reduction of power consumption is particularly beneficial for wireless applications, since it increases the battery life and decreases the energy that needs to be transferred.
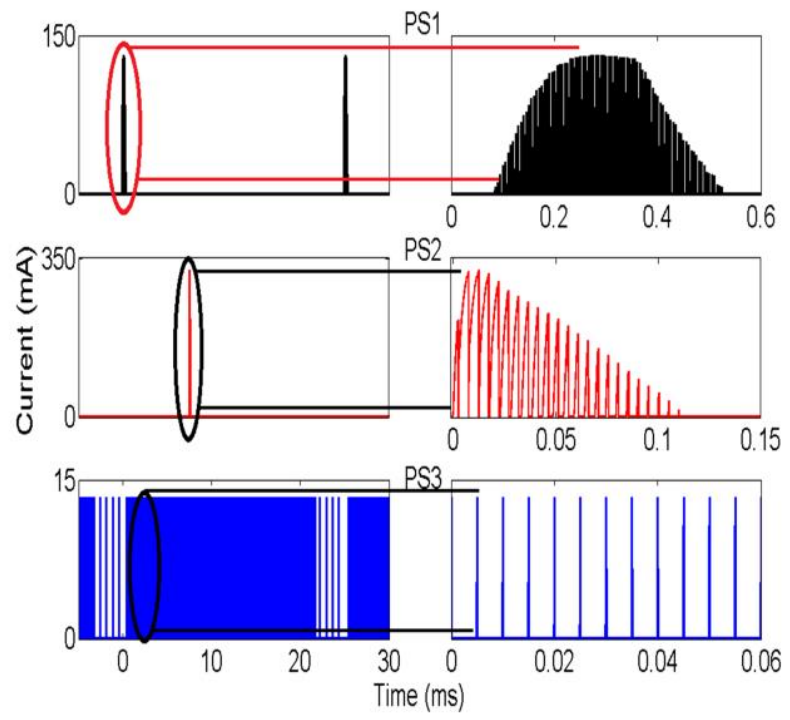

Fig 6. Current in the MOSFET in each strategy. Left: global picture; right zoom on switching period. Same remark as in Fig.5.

Different authors already reported DC/DC issues in portable stimulator designs ${ }^{13,17}$. Among the most common solutions is the flyback configuration. However, flyback circuits are usually bulky and expensive ${ }^{14,16,17}$. Another option is to use a booster, which does not require large components ${ }^{16,18}$, but does not usually either show a good efficiency. Despite this, there are currently many designs and forms of control proposed for DC-DC boost converters ${ }^{13,15}$. However, they are usually complex systems focusing on high levels of currents and voltages. For this reason, some stimulator designs rather use commercially available DC-DC converters ${ }^{18}$, which are usually expensive. This paper proposes a simple circuit, with a high efficiency, hence increasing the battery life. It uses the concept of pumps used in conjunction with a hysteresis-controlled boost converter. Very fast charge pump concept has already been used in implantable stimulation devices 19,20 , and it is used in high voltage for flash drivers in digital cameras, but the energy efficiency is low.

Since this strategy relies on the storage capacitor to provide the energy needed during stimulation, the value of the storage capacitor is higher than for traditional designs. This could lead to an increase in its size, which is a drawback since the available space is usually limited. However, the required value (here $3.5 \mu \mathrm{F}$ ) is still small enough to find commercially available capacitors that are of reasonable size. Also, the supply voltage of the output stage is now fluctuating above the nominal value, leading to higher voltages. This could be an issue if electronic components in the output stage do not support this increase in voltage. However, this increase is also governed by the value of the storage 
Table 1. Comparision between the stimulation systems.

\begin{tabular}{|ccc}
\hline & $\begin{array}{c}\text { Implanted } \\
\text { Gastrostimulator }\end{array}$ & $\begin{array}{c}\text { Foot drop } \\
\text { stimulator }\end{array}$ \\
\hline $\begin{array}{c}\text { Max. Current } \\
\text { amplitude for } \\
\text { Stimulation pulse }\end{array}$ & $5[\mathrm{~mA}]$ & $\begin{array}{c}100 \\
{[\mathrm{~mA}]}\end{array}$ \\
$\begin{array}{c}\text { Min. Supply Voltage } \\
\text { for the current source }\end{array}$ & $8[\mathrm{~V}]$ & $100[\mathrm{~V}]$ \\
\hline $\begin{array}{c}\text { Max. Load (including } \\
\text { electrodes) }\end{array}$ & $1600[\mathrm{Ohm}]$ & $\begin{array}{c}1000 \\
{[\mathrm{Ohm}]}\end{array}$ \\
$\begin{array}{c}\text { Stimulation Frequency } \\
\begin{array}{c}\text { Max. Stimulation pulse } \\
\text { width time }\end{array}\end{array}$ & $40[\mathrm{~Hz}]$ & $50[\mathrm{~Hz}]$ \\
\hline
\end{tabular}

capacitor. The value of the storage capacitor can therefore be increased to reduce fluctuations of the supply voltage, if needed. The future work will include the hardware implementation in both case studies.

\section{Acknowledgement}

This work was supported by the National Fund for Scientific and Technological Development, FONDECYT, Chile, through FONDECYT N 1140469

\section{Conflict of Interest}

The authors declare no potential conflict of interests.

\section{Corresponding Author}

P. Aqueveque, Biomedical Engineering, Electrical Engineering Department, Universidad de Concepción, Chile. E-mail: paaqueve@udec.cl

\section{E-mails of coAuthors}

Vicente Acuña: vacunaot@ulb.ac.be

Francisco Saavedra: frsaavedra@udec.cl

Adrien Debelle: addebell@ulb.ac.be

Laurent Lonys: 1lonys@ulb.ac.be

Nicolas Julémont: njulemon@ulb.ac.be

François Huberland: francois.huberland@ulb.ac.be Carmen Godfraind: carmen.godfraind@synergiam.com Antoine Nonclercq: anoncler@ulb.ac.be

\section{References}

1. Ghovanloo M, Najafi K. A small size large voltage compliance programmable current for biomedical implantable microstimulators. Proc 25th Annu Int Conf IEEE Eng Med Biol Soc IEEE Cat No03CH37439 2003;2:1979-82.

2. Mcclure M, Elder JS. Constant Input Power Modulation Technique for High Efficiency Boost Converter Optimized for Lithium-Ion Battery Applications Applied Power Electronics Conference and Exposition, 1996. APEC '96, Eleventh Annual, San Jose, CA, 1996, pp. 850-855 vol.2.
Table 2. Efficiency for the three strategies and the two case studies.

\begin{tabular}{ccc}
\hline & $\begin{array}{c}\text { Implanted } \\
\text { Gastrostimulator }\end{array}$ & $\begin{array}{c}\text { Foot drop } \\
\text { stimulator }\end{array}$ \\
\hline PS1 & $78.1 \%$ & $69.7 \%$ \\
PS2 & $47.3 \%$ & $69.5 \%$ \\
PS3 & $89.2 \%$ & $90.9 \%$ \\
\hline
\end{tabular}

3. Mohan R. Undeland, Power Electronics: Converters, Applications, and Design, 3rd Editio. 2002.

4. Liberson WT, Holmquest HJ, Scot D. Functional Electrotherapy in stimulation of peroneal nerve synchronyzed with swing phase of gait in hemiparetic patiens. Arch Phys Med Rehabil 1961:42:101-5.

5. Lonys L, Vanhoestenberghe A, Julémont N, et al., Silicone rubber encapsulation for an endoscopically implantable gastrostimulator. Med Biol Eng Comput 2015;53:4:319-29.

6. Drieskens S, Van der Heyden J, Demarest S, Tafforeau J. Is the different time trend (1997-2008) of the obesity prevalence among adults in the three Belgian regions associated with lifestyle changes? Arch Public Health, vol. 2014;72:18.

7. Dobbs R, Sawers C, Thompson F, et al. Overcoming obesity: An initial economic analysis. McKinsey Global Institute 2014.

8. $\mathrm{Ng} \mathrm{M}$, Fleming $\mathrm{T}$, Robinson $\mathrm{M}$, et al. Global, regional, and national prevalence of overweight and obesity in children and adults during 1980-2013: A systematic analysis for the Global Burden of Disease Study 2013," Lancet 2014;384-9945:766781.

9. Ibrahim M, Blero D, Deviere J. Endoscopic options for the treatment of obesity. Gastroenterology 2010;138:2228-32, 2232.e1.

10. Herron DM, Birkett DH, Thompson CC, et al. Gastric bypass pouch and stoma reduction using a transoral endoscopic anchor placement system: A feasibility study. Surg Endosc Other Interv Tech 2008;22:1093-9.

11. Griffith J, Daram S, Boatright B, et al. New Advances in Gastrointestinal Motility Research 2013;10. Springer Netherlands.

12. Lee EKF, Lam A. A Matching Technique for Biphasic Stimulation Pulse. IEEE International Symposium on Circuits and Systems ISCAS 2007;817-20.

13. Broderick B, Breen P, Ólaighin G. Electronic stimulators for surface neural prosthesis. J Autom Control 2008; 18:25-33. 
14. Ilic M, Vasiljevic D, Popovic DB. Programmable electronic stimulator for FES systems, IEEE Trans Rehabil Eng1994;2:234-9.

15. Simcox S, Davis G, Barriskill, et al. A portable, 8channel transcutaneous stimulator for paraplegic muscle training and mobility-A technical note. Journal of rehabilitation research and development, 2014;41-52.

16. Cheng KWE, Lu Y, Tong KYR, et al. Development of a Circuit for Functional Electrical Stimulation. IEEE Transactions on neural systems and rehabilitation engineering, 2004;12:43-7.

17. Thorsen R, Ferrarin M. Battery powered neuromuscular stimulator circuit for use during simultaneous recording of myoelectric signals. Med Eng Phys 2009;31:1032-7.

18. Qu H, Wang T, Hao M,et al. Development of a network FES system for stroke rehabilitation, Proc Annu Int Conf IEEE Eng Med Biol Soc EMBS 2011;3119-22.

19. Kar GC, Sawan M. Low-power high-voltage charge pumps for implantable microstimulators. ISCAS 2012 - IEEE Int Symp Circuits Syst 2012; 224750.

20. NadeauP, Sawan M. A flexible high voltage biphasic current-controlled stimulator. IEEE 2006 Biomed. Circuits Syst Conf Health Technol BioCAS 2006, 2006;206-9. 